\title{
Molecular Cytogenetic Characterization of Ciona intestinalis Chromosomes
}

\author{
Eiichi Shoguchi ${ }^{1{ }^{2 *}}$, Takeshi Kawashima ${ }^{2}$, Chizuko Nishida-Umehara ${ }^{3}$, \\ Yoichi Matsuda ${ }^{3}$ and Nori Satoh ${ }^{1,2}$ \\ ${ }^{1}$ CREST, Japan Science and Technology Agency, Kawaguchi, Saitama 332-0012, Japan \\ ${ }^{2}$ Department of Zoology, Graduate School of Science, Kyoto University, Sakyo-ku, \\ Kyoto 606-8502, Japan \\ ${ }^{3}$ Laboratory of Animal Cytogenetics, Center for Advanced Science and Technology, \\ and Laboratory of Cytogenetics, Division of Bioscience, Graduate School of \\ Environmental Earth Science, Hokkaido University, \\ Sapporo 060-0810, Japan
}

\begin{abstract}
The compact genome of the ascidian Ciona intestinalis has been sequenced. Chromosome karyotype and mapping of the genome sequence information on each of the 14 pairs of chromosomes are essential for genome-wide studies of gene expression and function in this basal chordate. Although the small chromosome size (most pairs measuring less than $2 \mu \mathrm{m}$ ) complicates accurate chromosome pairing based on morphology alone, the present results suggest that 20 chromosomes are metacentric and 8 are submetacentric or subtelocentric, and two pairs of large chromosomes (\#1 and \#2) were defined. The characterization of chromosomes by FISH and staining with propidium iodide indicated that $18 \mathrm{~S} / 28 \mathrm{~S}$ ribosomal gene repeats are present in the short arms of three pairs of chromosomes and that the short arms of these pairs show remarkable size polymorphism. In addition, each chromosome was characterized molecular cytogenetically by mapping representative BAC clones with FISH. The present study is therefore a first step in expanding the karyotype analysis and entire physical mapping of the genome sequence of Ciona intestinalis.
\end{abstract}

Key words: Ciona intestinalis, chromosomes, karyotype, rDNA, FISH, molecular cytogenetic characterization

\section{INTRODUCTION}

Ciona intestinalis is a urochordate ascidian that is collectable all year round throughout the world and that has therefore been intensively studied in various fields of zoology. Its position as a basal chordate in animal phylogeny makes it critical in studying the molecular mechanisms underlying the origin and evolution of chordates as well as vertebrates (Satoh, 2003; Azumi et al., 2003; Campbell et al., 2004). In addition, its tadpole-type larva represents the most simplified body plan of chordates, providing an experimental system for studying cellular and molecular mechanisms underlying the formation of the chordate body plan (Corbo et al., 2001; Satoh et al., 2003).

Our laboratory at Kyoto University has conducted a series of studies to promote Ciona intestinalis as an exper-

\footnotetext{
${ }^{*}$ Corresponding author. Phone: +81-75-753-4082;

Fax : +81-75-705-1113;

E-mail: eiichi@ascidian.zool.kyoto-u.ac.jp
}

imental material and/or system for future studies of developmental biology, evolutionary biology, and genome science (Satoh et al., 2003; Satoh, 2004). In collaboration with Ciona intestinalis cDNA consortium members, we conducted a large-scale expressed sequence tag (EST) analysis and cDNA project (Satou et al., 2002a, 2002b). To date, more than 680,000 ESTs have been deposited in the NCBI databank. In addition, full-insert sequence information has been obtained for the protein-coding region of more than 5,000 cDNAs (http://ghost.zool.kyoto-u.ac.jp/indexr1.html). The spatial expression of approximately 5,000 randomly selected genes was examined by whole-mount in situ hybridization (e.g., Satou et al., 2002b). Additionally, through a collaboration with the Joint Genome Institute (USA) and the National Institute of Genetics (Japan), the draft genome of Ciona intestinalis was sequenced and published in 2002 (Dehal et al., 2002). The approximately $160-\mathrm{Mb}$ genome is estimated to contain 15,852 protein-coding genes. Studies of Ciona genes indicated that some vertebrate gene families 
are found in a simplified form in Ciona (Dehal et al., 2002; Satou et al., 2003; Wada et al., 2003; Yamada et al., 2003; Yagi et al., 2003). This suggests that ascidians contain the basic ancestral complement of genes involved in cell signaling and development. In addition, the compact arrangement of developmentally relevant genes in the genome reveals that Ciona is an excellent model for a genome-wide survey of molecular mechanisms underlying specific gene expression required for formation of the chordate body (Harafuji et al., 2002; Keys et al., 2005).

However, the lack of genetic background information, a weakness shared by most marine invertebrates, makes it difficult to use the Ciona system for genome-scientific approaches in order to answer various biological questions. To overcome this problem, we used the approach of fluorescent in situ hybridization (FISH). In a previous study, we reported methodology for using FISH with Ciona intestinalis (Shoguchi et al., 2004). Following the previous success in using FISH, the present study aimed to develop the chromosome karyotype of Ciona intestinalis and their molecular cytogenetic characterization. Since a report by Colombera and Lazzaretto-Colombera (1978), showing that Ciona intestinalis has 14 pairs of chromosomes, no studies have characterized its karyotype.

\section{MATERIALS AND METHODS}

\section{Biological materials}

Ciona intestinalis adults were cultured at the Maizuru Fisheries Research Station of Kyoto University and the International Coastal Research Center, Ocean Research Institute, University of Tokyo. In this study, we used embryos at the neurula stage and early tailbud stage. Handling of the gametes and embryos was carried out as described by Shoguchi et al. (2004).

\section{Chromosome preparation and staining}

Chromosome preparation was modified from Shoguchi et al. (2004) as follows. After treatment with $0.04 \%$ colchicine (Sigma) for $30 \mathrm{~min}$, embryos were transferred to a $1.5-\mathrm{ml}$ microfuge tube and treated with hypotonic mixtures (seawater and $0.075 \mathrm{M} \mathrm{KCl}$ in ratios $2: 1$ and $1: 1$, for 5 min each). Fixation was performed in cold methanol:glacial acetic acid (3:1) fixative (chilled on ice) with three changes during $1 \mathrm{~h}$ and once after overnight (embryos were kept at $4^{\circ} \mathrm{C}$ ). The specimens were then stored in fixative at $-30^{\circ} \mathrm{C}$.

To prepare slides for the metaphase spreads, an excess $(x 3)$ of $60 \%$ acetic acid was added to a microfuge tube containing 10 20 embryos. One minute later, the embryos were pipetted gently onto pre-warmed $\left(48^{\circ} \mathrm{C}\right)$ ethanol-cleaned microscope slides $(60 \mu \mathrm{l}$ per slide) and left until dry (1-2 h). The FISH spreads were incubated at $65^{\circ} \mathrm{C}$ for $3 \mathrm{~h}$ in a clean incubator.

Metaphase chromosomes were stained with Giemsa or Hoechst 33258 and propidium iodide (PI). To observe Giemsa-stained spreads, the slides were stained for $1-2 \mathrm{~h}$ with $6 \%$ Giemsa solution (Merck) in 1/15 M phosphate buffer (pH 6.8). To observe Hoechst 33258 and PI stained spreads, the chromosome slides were stained for $5 \mathrm{~min}$ with $1 \mu \mathrm{g} / \mathrm{ml}$ Hoechst 33258 (Sigma), rinsed for $5 \mathrm{~min}$ briefly with $2 x S S C$, and then stained for $30 \mathrm{~min}$ with $0.5 \mu \mathrm{g} / \mathrm{ml}$ propidium iodide (Sigma).

\section{Fluorescent in situ hybridization (FISH) with BAC clones}

A library of BAC (bacterial artificial chromosome) clones with an average length of $135 \mathrm{~kb}$ was constructed from Ciona intestinalis sperm DNA (Kobayashi et al., 2002). To find BACs containing ribosomal genes, we searched the sequences similar to $C$. intestinalis 18S rRNA (Accession No. AB013017) and 28S rRNA (Accession No. AF212177) sequences in the Ghost Database (http:// ghost.zool.kyoto-u.ac.jp/indexr1.html) using the Blast algorithm. We found that $137 \mathrm{BAC}$ clones had sequences corresponding to $18 \mathrm{~S}$ rRNA and/or 28S rRNA (BLAST algorithm, probability<e-100) at both ends. Of them, we used probes derived from BAC clones GECi20_h11, GECi17_m21, GECi17_123, GECi30_115 and GECi41_e07. ad003p11 is a cDNA clone with a partial 18S rRNA sequence (http://ghost.zool.kyoto-u.ac.jp/indexr1.html).

We conducted a series of FISH analyses to map at least one BAC clone onto each of the 14 pairs of chromosomes. BAC clones corresponding to Scaffold 1 to 100 of C. intestinalis genome sequences (version 1) were selected as probes. Two-color FISH was performed as described previously (Shoguchi et al., 2004). Vectashield mounting medium with 4',6-diamidino-2-phenylindole (DAPI) (Vector Laboratories) was applied to the slides for chromosome counterstaining. Through these extensive analyses, we found that BAC clones (http://ghost.zool.kyoto-u.ac.jp/indexr1.html) GECi40_l15, GECi22_g17, GECi47_a17, GECi46_g06, GECi28_e05, GECi48_g08, GECi31_b20, GECi23_02, GECi30_d14, GECi30_f09, GECi28_m24, GECi21_a11, GECi31_b04, and GECi42_m08, covered all 14 pairs of chromosomes (Table 1).

\section{RESULTS AND DISCUSSION}

\section{Karyotype and $\mathrm{PI} /$ Hoechst 33258 staining}

We confirmed, by examination of more than 50 Giemsa-stained well-spread chromosome preparations, the results of a previous study by Colombera and LazzarettoColombera (1978) that Ciona intestinalis has 14 pairs of chromosomes. However, not every chromosome spread always contained exactly 28 chromosomes. Fig. 1 shows a representative karyotype of $C$. intestinalis. The specimen possesses 20 metacentric $(\mathrm{m})$ chromosomes and 8 submetacentric (sm) or subtelocentric (st) chromosomes (Levan et al., 1964). The characterization of chromosomes was not constant due to the size polymorphism in the short arms, as will be discussed later. Although the small chromsome size complicated accurate chromosome pairing based on morphology alone, it was noticed that amongst the 20 metacentric chromosomes, one pair was quite large; we named this chromosome \#1 (Fig. 1B). In addition, in the largest chromosome pair among the 8 submetacentric/subtelocentric chromosomes, the short arm was very small and showed morphological polymorphism (Fig. 1A; arrows); we named this chromosome \#2 (Fig. 1B). G-banding is usually used in higher vertebrates for unequivocal recognition of each chromosome pair. However, clear G-banding was not obtained in C. intestinalis. The C. intestinalis genome is AT-rich $(65 \%$ of the sequence) (Dehal et al., 2002) and is likely to lack typical GC-rich regions in the chromosomes.

Metaphase chromosome preparations were also stained with Hoechst 33258 and PI (Fig. 2A, B). We found that the short arms of six chromosomes were stained intensely with PI. However, presumably due to morphological polymorphism and some technical problems, sometimes the short arms of only four or five chromosomes were stai- 

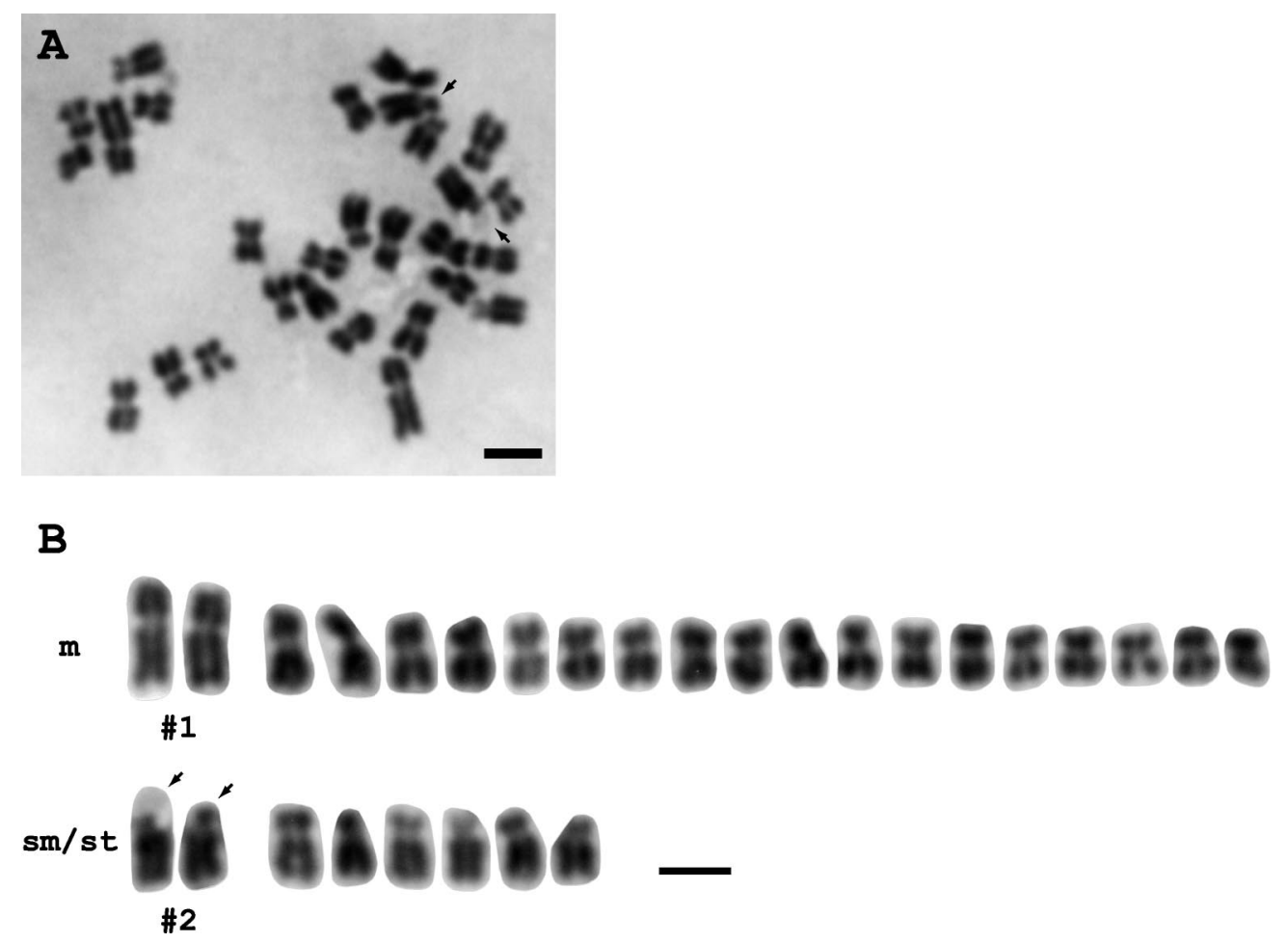

Fig. 1. Karyotype analysis of Ciona intestinalis chromosomes. (A) A preparation of metaphase chromosomes stained with Giemsa. Arrows indicate the very short arm of a pair of chromosomes (\#2). (B) The karyotype analysis. m, metacentric chromosome; sm/st, submetacentric or subtelocentric chromosome. The bars represent $2 \mu \mathrm{m}$.

ned intensely with $\mathrm{PI}$ (Fig. 2A, B) and those of one or two chromosomes were stained faintly (Fig. 2A, B; arrows). In fish chromosomes, it was reported that PI-positive blocks are nucleolus organizer regions (NORs) (Rab et al., 1996; Fischer et al., 2000). The draft sequence of the Ciona intestinalis genome indicates that $18 \mathrm{~S} / 28 \mathrm{~S}$ rDNA repeats are those of the highest copy number (Dehal et al., 2002). Thus, the PI-positive blocks may be regions containing rDNA repeats. To test this possibility, we tried to identify the chromosomal location of rDNA repeats by FISH.

\section{Identification of ribosomal DNA (rDNA)}

To clarify the locations of $18 \mathrm{~S} / 28 \mathrm{~S}$ rDNA repeats, we carried out FISH with the BAC clones (GECi20_h11, GECi17_m21, GECi17_I23, GECi30_115 and GECi41_e07) that contained ribosomal genes. Fig. $2 \mathrm{C}$ shows the hybridization with a rDNA-containing BAC clone (GECi20_h11; red and arrows), which indicated that rDNA regions are located on the short arms of three pairs of chromosomes. This result was confirmed by using the four other BAC clones with 18S/ 28S rDNA repeats (GECi17_m21, GECi17_I23, GECi30_I15 and GECi41_e07) (data not shown). ad003p11 is a cDNA clone containing a partial 18S rRNA sequence and this cDNA probe hybridized with similar regions of the chromosomes (data not shown). These results indicate that there are three rDNA clusters per haploid genome of $C$. intestinalis.

Fig. $2 \mathrm{C}$ also shows the hybridization of a rDNA-containing BAC clone (GECi20_h11) (red) and four different BAC probes without rDNA sequences (GECi47_a17, GECi46_g06, GECi28_e05 and GECi27_g06) (green). GECi47_a17, GECi46_g06 and GECi28_e05 are located on different pairs of chromosomes (Table 1). GECi47_a17 and GECi27_g06 are located on the same arm of a chromosome (Table 1). As shown in Fig. 2C, GECi47_a17, GECi46_g06, GECi28_e05 and GECi27_g06 are landmarks in the long arms of rDNAbearing chromosomes.

In addition, after FISH with the BAC clone, counterstaining with $\mathrm{PI}-\mathrm{Hoechst} 33258$ was performed, and the findings indicated that PI-positive blocks correspond to rDNA regions (Fig. 2D, E; red arrows). Therefore, PI/ Hoechst 33258 staining is efficient for detecting rDNA clusters. We also noticed that the pair of the rDNA-bearing chromosomes might have size polymorphisms in the short arm (Fig. 2D, E; white arrows); such size polymorphism occurs in most vertebrates species (e.g., Galetti et al., 1995; Fujiwara et al., 1998).

\section{Molecular cytogenetic characterization of Ciona intesti- nalis chromosomes}

We were able to identify chromosomes \#1 and \#2 based on morphology and to distinguish three pairs of chromosomes with $18 \mathrm{~S} / 28 \mathrm{~S}$ ribosomal genes. In addition, two pairs of submetacentric/subtelocentric chromosomes were distinguished by staining with $\mathrm{Pl} /$ Hoechst 33258 (Fig. 2B). However, because it was difficult to identify chromosomes exactly using these methods, we attempted characterization of every chromosome using a molecular cytogenetic method. As mentioned in the Methods section, we are now 


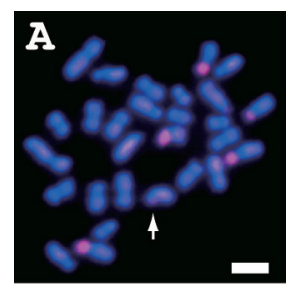

B
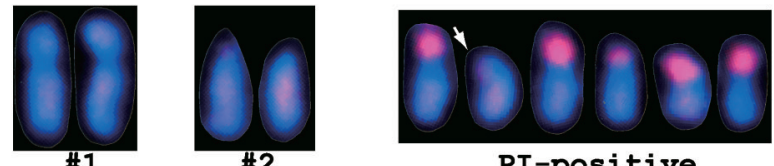

\#1

\#2

PI-positive

m

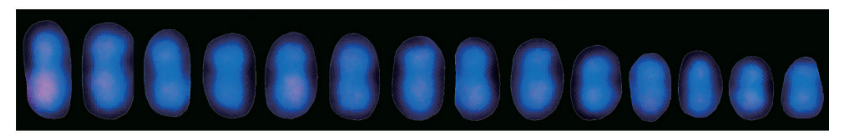

$\mathrm{sm} / \mathrm{st}$
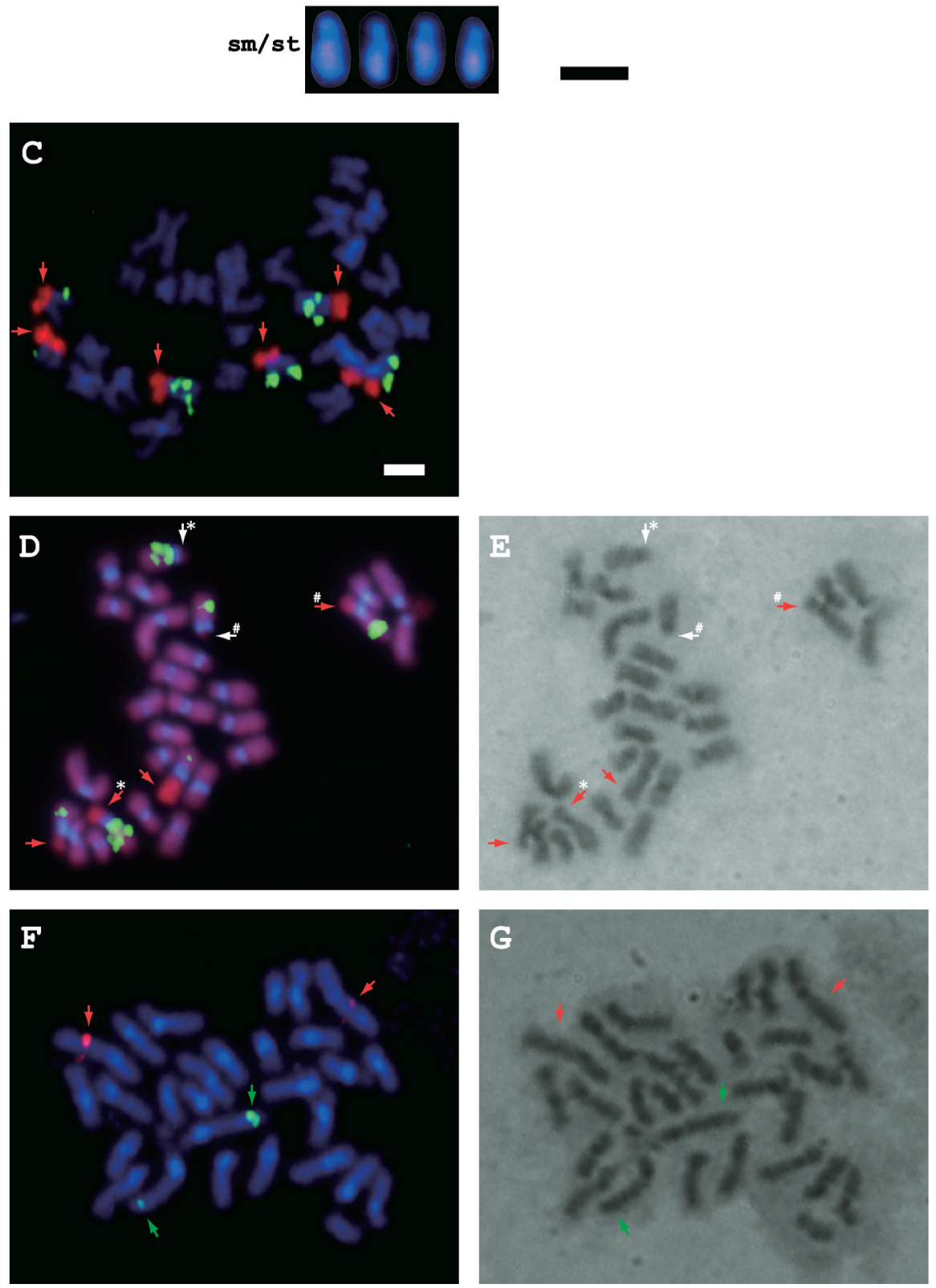

Fig. 2. Propidium iodide (PI) and Hoechst 33258 staining of Ciona intestinalis chromosomes and FISH with specific BAC probes. (A) Metaphase chromosomes stained with Hoechst 33258 (blue) and PI (red), and (B) the karyotype analysis. The short arms of five chromosomes are stained intensely with $\mathrm{PI}$ and one is stained faintly (arrow), suggesting the presence of 18S/28S rDNA clusters. (C) Two-color FISH with a BAC probe (GECi20_h11; red) containing the 28S rDNA sequence and four BAC probes (GECi47_a17, GECi27_g06, GECi46_g06 and GECi28_e05; green) without rDNA sequences. The slide was counterstained with DAPI. Arrows indicate the short arms of three pairs of chromosomes stained with GECi20_h11 (red). (D, E) FISH with GECi47_a17 (green), GECi27_g06 (green), GECi46_g06 (green) and GECi28_e05 (green), counterstained with $\mathrm{PI} /$ Hoechst 33258 (D). Red arrows indicate four short arms stained with PI/Hoechst 33258, while white arrows indicate two short arms with faint staining due to morphological polymorphism. (E) The specimen stained with Giemsa solution following the FISH observation. \# and * indicate two pairs of chromosomes identified with FISH (D). (F) FISH with GECi40 I15 (red) and GECi22 g17 (green) and then stained with Giemsa solution $(G)$. Red arrows indicate chromosomes stained with GECi40_115 probe and green arrows those stained with GECi22_g17 probe. The chromosomes with arrows are large chromosomes (\#1 and \#2). Bars represent $2 \mu \mathrm{m}$. 
Table 1. BAC clones used as probes to characterize Ciona intestinalis chromosomes

\begin{tabular}{lll}
\hline chromosome type $^{1)}$ & BAC clone mapped & \\
\hline $\mathrm{m}$ (\#1) & GECi40_115 (Scaffold_23) & \\
$\mathrm{sm} / \mathrm{st}(\# 2)^{*}$ & GECi22_g17 (Scaffold_15) & \\
$\mathrm{sm} / \mathrm{st}^{*}$ & GECi47_a17(Scaffold_6) & GECi20_h11(rDNA) \\
& GECi27_g06(Scaffold_44, & \\
& Scaffold_836) & \\
$\mathrm{m}^{*}$ & GECi46_g06(Scaffold_43) & GECi20_h11(rDNA) \\
$\mathrm{m}^{*}$ & GECi28_e05(Scaffold_19) & GECi20_h11(rDNA) \\
$\mathrm{sm} / \mathrm{st}$ & GECi48_g08 (Scaffold_10) & \\
$\mathrm{sm} / \mathrm{st}$ & GECi31_b20 (Scaffold_16) & \\
$\mathrm{m}$ & GECi23_j02 (Scaffold_20) \\
$\mathrm{m}$ & GECi30_d14 (Scaffold_4) \\
$\mathrm{m}$ & GECi30_f09 (Scaffold_7) \\
$\mathrm{m}$ & GECi28_m24 (Scaffold_41) & \\
$\mathrm{m}$ & GECi21_a11 (Scaffold_35) & \\
$\mathrm{m}$ & GECi31_b04 (Scaffold_79) \\
$\mathrm{m}$ & GECi42_m08 (Scaffold_31) & \\
\hline
\end{tabular}

1) $\mathrm{m}$, metacentric; sm/st, submetacentric or subtelocentric (Levan et al., 1964). ${ }^{*}$ These chromosomes show remarkable polymorphism.

2) Scaffolds corresponding to the BAC clones are shown in parenthesis.

conducting a series of FISH analyses to map at least one BAC clone onto each of the 14 pairs of chromosomes. As a result, we found that BAC clones GECi40_115, GECi22_g17, GECi47_a17, GECi46_g06, GECi28_e05, GECi48_g08, GECi31_b20, GECi23_j02, GECi30_d14, GECi30_f09, GECi28_m24, GECi21_a11, GECi31_b04, and GECi42_m08 are each mapped onto a different chromosome (Table 1). Although the chromosome types listed in Table 1 are based on the morphology of the FISH counterstained image, they also correspond to about the Giemsa-stained morphology (Fig. 2F, G; arrows). Therefore, each of the Ciona intestinalis chromosomes is now distinguishable using the landmark of a BAC clone. Fig. 2F and 2G indicate that GECi40_I15 (red) and GECi22_g17 (green) are mapped on one pair of long chromosomes each (red and green arrows in Fig. 2F, $\mathrm{G})$. Giemsa staining following FISH observation confirmed that these chromosomes correspond to \#1 and \#2 based on morphology alone. Altogether, these results indicate that GECi40_115 and GECi22_g17 are mapped on \#1 and \#2, respectively. Thus, in the near future, unequivocal recognition of homologous pairs should be facilitated by these chromosome-specific markers.

\section{CONCLUSION}

In this study, we characterized 14 pairs of chromosomes in Ciona intestinalis using molecular cytogenetic analyses. This information provides us indicators for use in further mapping of genome sequence information onto the chromosomes. Since mapping of genes onto chromosomes is essential for further studies of genome-wide control of the expression of developmentally relevant genes, the present results will further promote Ciona intestinalis to be an appropriate system for future studies in zoology.

\section{ACKNOWLEDGMENTS}

We wish to thank Dr. Yutaka Satou for his enormous help in the preparation of BAC sequence information, and Makoto Hamaguchi for the analysis of genome sequences. We also thank Dr. Takahito Nishikata and Dr. Shungo Kano for useful discussions and Chikako Imaizumi for her support in the maintenance of sequence information in our laboratory. Kazuko Hirayama, Maizuru Fisheries Research Station and International Coastal Research Center are acknowledged for their help in culturing of Ciona intestinalis. The present study was supported in part by a Grant-in-Aid from MEXT, Japan to NS (12202001).

\section{REFERENCES}

Azumi K, De Santis R, De Tomaso A, Rigoutsos I, Yoshizaki F, Pinto MR, Marino R, Shida K, Ikeda M, Ikeda M, Arai M, Inoue Y, Shimizu T, Satoh N, Rokhsar DS, Du Pasquier L, Kasahara M, Satake M, Nonaka M (2003) Genomic analysis of immunity in a urochordate and the emergence of the vertebrate immune system: "waiting for Godot". Immunogenetics 55: 570-581

Campbell RK, Satoh N, Degnan BM (2004) Piecing together evolution of the vertebrate endocrine system. Trends Genet 20:359366

Colombera D, Lazzaretto-Colombera I (1978) Chromosome evolution in some marine invertebrates. In "Marine Organisms" Ed by B Battaglia et al., Plenum, New York, pp 487-525

Corbo JC, Di Gregorio A, Levine M (2001) The ascidian as a model organism in developmental and evolutionary biology. Cell 106: 535-538

Dehal P, Satou Y, Campbell RK et al. (2002) The draft genome of Ciona intestinalis: insights into chordate and vertebrate origins. Science 298: 2157-2167

Fischer C, Ozouf-Costaz C, Roest Crollius H, Dasilva C, Jaillon O, Bouneau L, Bonillo C, Weissenbach J, Bernot A (2000) Karyotype and chromosome location of characteristic tandem repeats in the pufferfish Tetraodon nigroviridis. Cytogent Cell Genet 88: 50-55

Fujiwara A, Abe S, Yamaha E, Yamazaki F, Yoshida MC (1998) Chromosomal localization and heterochromatin association of ribosomal RNA gene loci and silver-stained nucleolar organizer regions in salmonid fishes. Chrom Res 6: 463-471

Galetti PM Jr, Mestriner CA, Monaco PJ, Rasch EM (1995) Postzygotic modifications and intra- and inter-individual nucleolar organizing region variations in fish: report of a case involving Leporinus friderici. Chrom Res 3: 285-290

Harafuji N, Keys DN, Levine M (2002) Genome-wide identification of tissue-specific enhancers in the Ciona tadpole. Proc Natl Acad Sci USA 99: 6802-6805

Keys DN, Lee B-i, Di Gregorio A, Harafuji N, Detter C, Wang M, Kahsai O, Ahn S, Zhang C, Doyle S, Satoh N, Satou Y, Saiga H, Christian A, Rokhsar D, Hawkins T, Levine M, Richardson P (2005) A saturation screen for cis-acting regulatory DNA in the Hox genes of Ciona intestinalis. Proc Natl Acad Sci USA 102: 679-683

Kobayashi M, Matsuda M, Asakawa S, Shimizu N, Nagahama Y, Satou Y, Satoh N (2002) Construction of BAC libraries derived from the ascidian Ciona intestinalis. Genes Genet Syst 77: 283-285

Levan A, Fredga K, Sandberg AA (1964) Nomenclature for centromeric position on chromosomes. Hereditas 52: 201-220

Rab P, Reed KM, Ponce de Leon FA, Phillips RB (1996) A new 
method for detecting nucleolus organizer regions in fish chromosomes using denaturation and propidium iodide staining. Biotech Histochem 71: 157-162

Satoh N (2003) The ascidian tadpole larva: Comparative molecular development and genomics. Nature Rev Genet 4: 285-295

Satoh N (2004) Genomic resources for ascidians: Sequence/ expression databases and genome projects. In "Methods in Cell Biology. Experimental analysis of the development of sea urchins and other non-vertebrate deuterostomes Vol 74" Ed by CA Ettensohn et al., Elsevier Inc., Amsterdam, pp 759-774

Satoh N, Satou Y, Davidson B, Levine M (2003) Ciona intestinalis: an emerging model for whole-genome analyses. Trends Genet 19: $376-381$

Satou Y, Yamada L, Mochizuki Y, Takatori N, Kawashima T, Sasaki A, Hamaguchi M, Awazu S, Yagi K, Sasakura Y, Nakayama A, Ishikawa $\mathrm{H}$, Inaba K, Satoh N (2002a) A cDNA resource from the basal chordate Ciona intestinalis. Genesis 33: 153-154

Satou Y, Takatori N, Fujiwara S, Nishikata T, Saiga H, Kusakabe T, Shin-i T, Kohara Y, Satoh N (2002b) Ciona intestinalis cDNA projects: expressed sequence tag analyses and gene expression profiles during embryogenesis. Gene 287: 83-96

Satou Y, Imai KS, Levine M, Kohara Y, Rokhsar D, Satoh N (2003) A genomewide survey of developmentally relevant genes in Ciona intestinalis: I. Genes for bHLH transcription factors. Dev Genes Evol 213: 213-221
Shoguchi E, Ikuta T, Yoshizaki F, Satou Y, Satoh N, Asano K, Saiga $\mathrm{H}$, Nishikata $\mathrm{T}$ (2004) Fluorescent in situ hybridization to ascidian chromosomes. Zool Sci 21: 153-157

Wada S, Tokuoka M, Shoguchi E, Kobayashi K, Di Gregorio A, Spagnuolo A, Branno M, Kohara Y, Rokhsar D, Levine M, Saiga H, Satoh N, Satou $Y$ (2003) A genomewide survey of developmentally relevant genes in Ciona intestinalis: II. Genes for homeobox transcription factors. Dev Genes Evol 213: 222234

Yagi K, Satou Y, Mazet F, Shimeld SM, Degnan B, Rokhsar D, Levine M, Kohara Y, Satoh N, (2003) A genomewide survey of developmentally relevant genes in Ciona intestinalis: III. Genes for Fox, ETS, nuclear receptors and NFкB. Dev Genes Evol 213: $235-244$

Yamada L, Kobayashi K, Degnan B, Satoh N, Satou Y (2003) A genomewide survey of developmentally relevant genes in Ciona intestinalis: IV. Genes for HMG transcriptional regulators, bZip and GATA/Gli/Zic/Snail. Dev Genes Evol 213: 245-253

(Received December 29, 2004 / Accepted March 2, 2005) 